This report was prepared as an account of work sponsored by an agency of the United States Government. Neither the United States Government nor any agency thereof, nor any of their employees, makes any warranty, express or implied, or assumes any legal liability or responsibility for the accuracy, completeness, or usefulness of any information, apparatus, product, or process disclosed, or represents that its use would not infringe privately owned rights. Reference herein to any specific commercial product, process, or service by trade name, trademark, manufacturer, or otherwise does not necessarily constitute or imply its endorsement, recommendation, or favoring by the United States Government or any agency thereof. The views and opinions of authors expressed herein do not necessarily state or reflect those of the United States Government or any agency thereof.

$$
\text { SAND- }-95-0706 C
$$

\title{
CHARACTERIZATION AND REDUCTION OF PROMPT ELECTRICAL NOISE ON THE SATURN PRS X-RAY SIMULATOR
}

\author{
D.E. Johnson ${ }^{*}$, F.W. Davies ${ }^{*}$, and M.A. Hedemann ${ }^{* *}$ \\ * Ktech Corporation, 901 Pennsylvania NE, Albuquerque, NM 87110 \\ ** Sandia National Laboratories, P.O. Box 5800, Albuquerque, NM 87185
}

Experiments have been conducted to identify and characterize the source of prompt electrical noise observed on piezoelectric sensor data traces recorded at the Saturn soft $x$-ray source. The amplitude of this noise spike often exceeded the amplitude of the signal of interest. Although the noise duration was short (50 to $100 \mathrm{~ns}$ ), it interfered with many measurements of soft $\mathrm{x}$-ray induced material response detected with piezoelectric gauges. The noise spike can be due to a combination of sources, including bremsstrahlung radiation. However, it was determined that high-energy electrons generated about 90 per cent of the recorded signal in the near-source region. Fixtures employing permanent magnets reduced the noise spike amplitude by more than a factor of ten, permitting sensitive, high-fidelity, material response measurements to be made with greater accuracy and confidence. Furthermore the test environment is more accurately controlled and defined.

\section{INTRODUCTION}

The Saturn $x$-ray simulator [1] at Sandia National Laboratories, New Mexico can be configured as a Plasma Radiation Source (PRS) to generate soft $x-$ rays of 1 to $5 \mathrm{keV}$ energy [2]. In this configuration, Saturn is used to determine material or component response to pulsed $x$-ray exposure of 0.1 to 50 $\mathrm{cal} / \mathrm{cm}^{2}$. Material response is determined primarily by measuring the $x$-ray generated stress pulse that propagates through the sample using piezoelectric stress gauges [3] or VISAR. Until recently, the use of piezoelectric stress gauges has been complicated by the presence of a large noise spike that occurs coincident with $\mathrm{x}$-ray generation. As Figure 1 shows, the amplitude of this noise spike often exceeded that of the signal of interest, especially when the machine used an argon gas puff as the source. The high-amplitude noise spike, though brief in duration and proceeding the stress pulse, often drove the recording amplifiers into saturation and either obscured the signal of interest or shifted the baseline enough to prevent accurate measurement. It is also important to note that the noise spike was only large when the machine used a gas puff, such as argon, as the source. The use of wire arrays, such as aluminum, resulted in much lower noise spike amplitudes that seldom interfered with material response measurement.

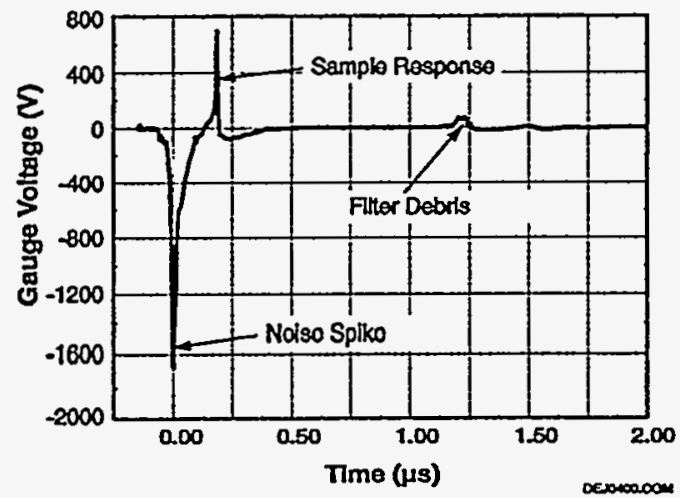

FIGURE 1. The noise spike amplitude often interfered with the signal of interest.

Work supported by Sandia National Laboratories, Albuquerque, NM, under contract AF-8570

This work was supported by the United States Department of Energy under Conirict DE-ACD4-94ALB5000.

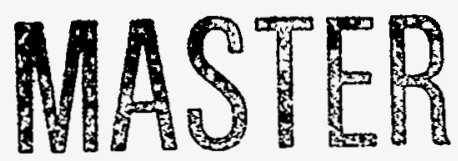




\section{DISCLAIMER}

Portions of this document may be illegible in electronic image products. Images are produced from the best available original document. 
Since Saturn in the mode of interest delivers a pulse of about $400 \mathrm{~kJ}$ of electrical energy to the diode at $2 \mathrm{MV}$ and $8 \mathrm{MA}$, electrical noise in not unexpected. Potential noise sources include electromagnetic pulse (EMP), electrons, and bremsstrahlung radiation. A variety of techniques, including shielding, grounding, baffles, and permanent magnets, were used separately and in combination to reduce the noise spike amplitude to an acceptable level. The single most effective tool was the use of permanent magnets to sweep out highenergy electrons. These electrons were found to generate 90 to 95 percent of the observed noise signal on $x$-cut quartz gauges.

\section{NOISE REDUCTION TECHNIQUES}

Noise reduction techniques commonly used in pulsed power environments are routinely applied at Saturn. These include various forms of shielding, grounding, and baffles. Recently, 0.1 to $0.4 \mathrm{~T}$ permanent magnets have been added to the test fixtures and proven extremely effective at reducing the noise spike amplitude. The following paragraphs describe several experiments that lead us to conclude that high-energy electrons were generating most of the electrical noise.

Differential Absorption Spectrometer (DAS)

This diagnostic was originally designed to measure photon spectra of the Saturn 1-2 $\mathrm{MeV}$ bremsstrahlung sources. The DAS consists of 13 differentially filtered thermoluninescent dosimeters (TLDs), where ratios of TLD doses are normally used to estimate photon spectral content. To obtain an estimate of either photon or electron spectral content, the DAS was fielded at a $0.05 \mathrm{cal} / \mathrm{cm}^{2}$ fluence level of $3.1 \mathrm{keV} x$-rays. Post-shot analysis indicated either electron energies peaked in the 0.4 $2.0 \mathrm{MeV}$ range (typically $-0.7 \mathrm{MeV}$ ), or photon energies peaked in the $20-30 \mathrm{keV}$ range. While the DAS could not be used a priori to differentiate between photons and electrons, the measured TLD doses ( $400 \mathrm{~Gy}$ ) were inconsistent with sourcestrength capabilities in the $20-30 \mathrm{keV}$ photon energy range. These results therefore strongly indicated a large electron-based component to the noise.

Shielding

Enclosing the sensor in metal shielding serves two functions: capacitive, magnetic, and radiated electrical noise are blocked or reduced by the Faraday enclosure; and noise induced by soft $x$-rays and electrons can be reduced. The effectiveness of the shielding depends primarily on the material properties, atomic number, conductivity, permeability, and density, and on the thickness of material used.

An experiment was conducted at Saturn using two shielding materials, aluminum and stainless steel, in several different thicknesses. Nominally identical $x$ cut quartz gauges housed in aluminum canisters were fielded with the sample shielding material bonded onto the front surface of the quartz element. The gauges were placed such that the sample was normal to the radiation path and directly between the source and the gauge. All samples were attached with conductive silver epoxy around the edges forming a complete Faraday enclosure.

As Figure 2 shows, the noise spike amplitude decreased in a roughly linear manner as the areal density of the sample was increased. Also, an equal thickness of stainless steel was about six times more effective than aluminum, even though ahuminum is a much better electrical conductor. The dependence on areal density, rather than electrical conductivity, indicates that the electrical noise spike is probably generated by penetrating electrons and not by pulsed power noise that is electrically coupled into the sensor.

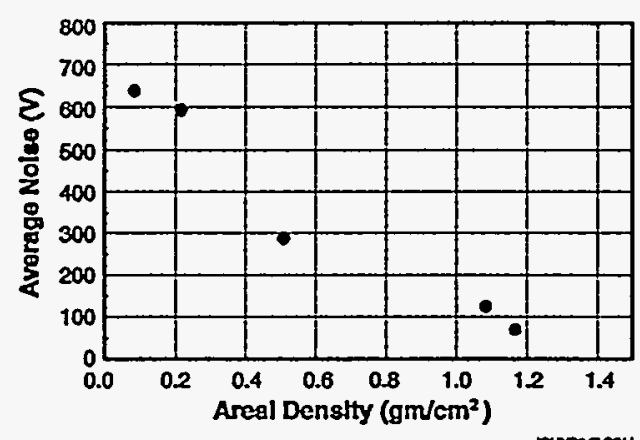

FIGURE 2. Saturn noise spike amplitude vs areal density of shielding.

The maximum penetration range, $R$ in $\mathrm{gm} / \mathrm{cm}^{2}$, of electrons in both ahuminum and stainless steel depends linearly on the electron energy, $\mathrm{E}$ in $\mathrm{MeV}$ [4]: 


$$
\mathrm{R}=0.542 \mathrm{E}-0.133
$$

This equation is accurate for electron energies from about $700 \mathrm{keV}$ to $3 \mathrm{MeV}$. Below $700 \mathrm{keV}$ it begins to overestimate the range, but still provides a reasonable approximation.

If we assume that the noise spike amplitude was proportional to the number of electrons that penetrated the shield layer, we can use Equation 1 to estimate the electron energies. As shown in Figure 2 , the noise amplitude was reduced 60 per cent by $0.55 \mathrm{gm} / \mathrm{cm}^{2}$ of aluminum, so many of the electrons were of energy $1.2 \mathrm{MeV}$ or less. Significant noise levels were still observed with $1.18 \mathrm{gm} / \mathrm{cm}^{2}$ of material, so some electron energies may have been as high as $2.4 \mathrm{MeV}$. This range of energies is consistent with observation based on the DAS analysis, assuming the source of the gauge noise and of the DAS activation energy was both electrons.

\section{Orientation}

The penetrating radiation or electrons that were hypothesized as the noise source appeared to originate from the plasma radiation source (PRS) region or very close to it. The shielding samples used in the experiment just described were placed between the $x$-ray source and the sensor. The samples were just large enough to cover the crystal and epoxy potting compound and would therefore block only $x$-rays and electrons that originated at the source.

In a second experiment, gauges were oriented such that the sensors were pointed away from the source. These gauges had much lower noise spike amplitudes than identical gauges pointed at the source. This second experiment supported the hypothesis that the noise was generated by $x$-rays or electrons that originated within or from very near the PRS.

\section{Permanent Magnets}

None of the previously described experiments could definitively differentiate between electrons and $X$-rays as the source of the noise spike energy, so an experiment using permanent magnets was designed. $\mathrm{X}$-rays are unaffected by magnetic fields, but if the noise source was electrons, the magnets would sweep out or trap many of the electrons and reduce the noise amplitude. The permanent magnets were arranged to prochuce a field transverse to the electron flight path. As the electrons traveled through the magnetic field, the Lorentz force would deflect the electrons or trap them in spiral orbits, depending on the electron energy (velocity) and the field strength. Figure 3 shows this arrangement schematically. Three fixtures were installed for each test. One fixture generated a field of approximately $0.1 \mathrm{~T}$, the second fixture generated a $0.4 \mathrm{~T}$ field, and the third, with no magnetic field, was used as a control. Calculations showed that the $0.1 \mathrm{~T}$ field would trap electrons of energy $100 \mathrm{keV}$ or less and sweep electrons of energy. $1 \mathrm{MeV}$ or less. The $0.4 \mathrm{~T}$ field would trap electrons of energy up to $1 \mathrm{MeV}$ and sweep electrons of energy $10 \mathrm{MeV}$ or less. Each of the three fixtures placed the gauges at the same range from the source and included a stainless steel aperture such that the gauges were exposed only to radiation and electrons emitted directly from the source region.

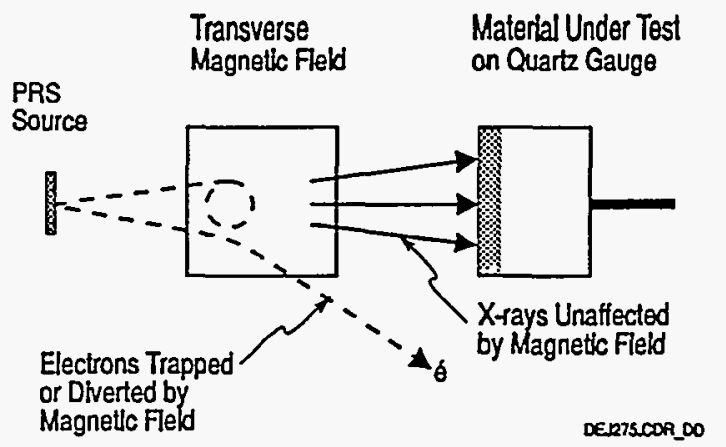

FIGURE 3. Permanent magnet fixtures for trapping or sweeping electrons.

Figure 4 shows the gauge records from one of two shots using the permanent magnet test fixtures. The noise spike amplitude was much lower on the two gauges fielded behind the magnetic fields. The test was repeated on a second shot with the same results as listed in Table 1.

The noise amplitude without magnets varied from 265 to $500 \mathrm{~V}$. The $0.1 \mathrm{~T}$ field reduced the noise amplitude by a factor of 10 to an average value of 32 $\mathrm{V}$. The $0.4 \mathrm{~T}$ field was even more effective, reducing the noise amplitude by 27 times to an average of $14 \mathrm{~V}$.

The strong effect observed with the magnetic fields confirms that the noise spike is caused primarily by high-energy electrons produced at the source. The small remaining noise level may be due to bremsstrahlung radiation. 


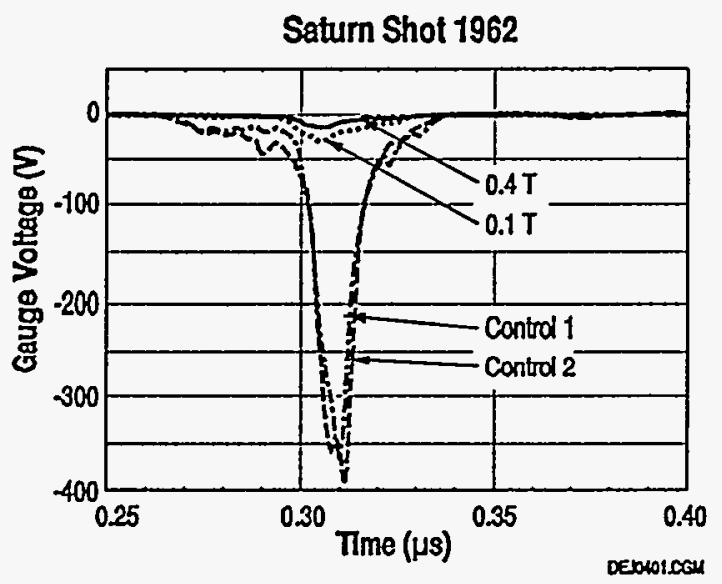

FIGURE 4. Noise spike records from Satum tests with permanent magnets.

\begin{tabular}{||c|c|c|c|c||}
\hline \multicolumn{4}{|c|}{$\begin{array}{l}\text { TABLE 1. Noise Amplitude Comparison with } \\
\text { Magnetic Fields (Noise Peak Amplitude in Volts) }\end{array}$} \\
\hline \hline & \multicolumn{2}{|c|}{ No Field Control } & \multicolumn{2}{|c|}{ Magnetic Field } \\
\cline { 2 - 5 } Shot & 1 & 2 & $0.1 \mathrm{~T}$ & $0.4 \mathrm{~T}$ \\
\hline \hline 1961 & 265 & 500 & 34 & 12 \\
\hline 1962 & 394 & 362 & 30 & 15 \\
\hline \hline Avg: & \multicolumn{2}{|c|}{380} & 32 & 14 \\
\hline
\end{tabular}

\section{DISCUSSION OF RESULTS}

The results of the experiments described here lead us to conclude that the noise spike observed at Saturn in gas-puff PRS mode is due almost entirely to energetic electrons emitted from the source region. These electrons range in energy from a few hundred $\mathrm{keV}$ up to about $2 \mathrm{MeV}$. Magnetic fields of 0.1 to $0.4 \mathrm{~T}$ over a volume of a few cubic centimeters are effective at trapping or sweeping out these electrons and reducing the noise spike amplitude by 10 to 30 times.

The relative absence of the noise spike when using a wire source array provides some clues as to the origin of the noise-generating electrons. We hypothesize that the electrons are generated in the gas puff source just before the gap breaks down to form the plasma channel that becomes the $x$-ray source. At this instant, before the gap breaks down and current flow begins, the gap voltage is several megavolts and the electric fields are oriented such that electrons would tend to be accelerated radially outward towards the gauges. In contrast, the electric fields are lower in a wire source because conduction begins immediately and is only limited by the source inductance and the relatively low resistance of the source wire array.

\section{CONCLUSIONS}

Permanent magnets can be used in Saturn and other PRS simulators to sweep out electrons and provide a test environment that is electrically much quieter. More sensitive and accurate material response measurements can be made and the test environment is better defined. Effective shielding design for both electrical and fiber-optic experiments has been simplified by this noise source identification and characterization.

\section{REFERENCES}

[1] Choate, I. M., The Saturn X-Ray Simulation Facility, A Technical and Logistical Information Guide for Users, Albuquerque, Sandia National Laboratories, 1989.

[2] Spielman, R. B., "Z-Pinched Experiments on Saturn at 30 TW", AIP Conference Proceedings No. 195, Dense Z-Pinches, 1989, pp 3-16.

[3] Johnson, D. E., Lee, I.M., Hedemann, M. A., and F. Bauer, "PVDF Measurement of Soft X-Ray Induced Shock and Filter Debris Impulse," Proceedings of the Conference on Shock Compression of Condensed Matter, 1993, pp. 1911-1914.

[4] B. T. Price, C. C. Horton, and K. T. Spinney, Radiation Shielding, Pergamon Press, 1957, pp. 71-72.

\section{ACKNOWLEDGEMENTS}

The authors are especially thankful for the technical assistance provided by C. DeLaCruz, R. Klingler, S. Heyborne, and M. Rice in performing these experiments and preparing the manuscript. 